\title{
Performance of a twin position-sensitive Frisch-grid ionization chamber for photofission experiments
}

\author{
Marius Peck ${ }^{1, *}$, Uwe Bonnes ${ }^{1}$, Joachim Enders $^{1}$, Alf Göök ${ }^{2}$, Joerg Hehner $^{3}$, and Stephan Oberstedt $^{2}$ \\ ${ }^{1}$ Institut für Kernphysik, Technische Universität Darmstadt, Schlossgartenstraße 9, D-64289 Darmstadt, Germany \\ ${ }^{2}$ European Commission, Joint Research Centre, Retieseweg 111, B-2440 Geel, Belgium \\ ${ }^{3}$ GSI Helmholtzzentrum für Schwerionenforschung GmbH, Planckstraße 1, D-64291 Darmstadt, Germany
}

\begin{abstract}
A position-sensitive twin Frisch-grid ionization chamber has been constructed for future photofission experiments using nearly monochromatic, linearly polarized gamma-ray beams. By exchanging the anode plates in the standard ionization chamber on both sides by an array of grid- and strip-anodes, which are rotated by $90^{\circ}$ relative to each other and read out by means of resistive charge division, a position sensitivity is achieved that allows the azimuthal fragment emission angle and hence the fission axis orientation to be determined. The performance of this gaseous detector has been studied using the well-known ${ }^{252} \mathrm{Cf}$ spontaneous fission process. The fission axis orientation could be determined relative to an arbitrary axis in space with a resolution better than $7^{\circ}$ FWHM. Measured pre-neutron mass and total kinetic energy distributions are consistent with literature, which ensures that the mass and energy resolution for fission fragments is not affected by the position-sensitive structure.
\end{abstract}

\section{Introduction}

Twin Frisch-grid ionization chambers (FGIC) [1] for the study of fission-fragment properties have been established as accurate and reliable detectors in the last decades. Fission-fragment mass and total kinetic energy distributions are determined using the double kinetic energy technique, based on the conservation of mass and linear momentum in the process of fission. The polar angle of the collinear fission fragments is determined from the time that free electrons, created by decelerating fission fragments in the counting gas, need to drift from the location of their creation to the anode plates [2]. Recently, a positionsensitive twin FGIC for fission fragment and prompt neutron correlation experiments was designed by Göök et al. [3]. By exchanging the anode plates in the standard chamber on both sides by an array of grid- and strip-anodes, which are rotated by $90^{\circ}$ relative to each other and read out by means of resistive charge division [4], a position sensitivity is achieved that allows the azimuthal fragment emission angle to be determined, too.

This manuscript reports on the investigation of a newly constructed position-sensitive twin FGIC chamber for future photofission experiments that may be used with nearly monochromatic, fully polarized gamma-ray beams from the $\mathrm{HI} \gamma \mathrm{S}$ facility at TUNL and ELI-NP. Since polarized photons are expected to yield strong azimuthal asymmetries in the fission fragment distribution, linked to the multipole character of the relevant excitations (see Ref. [5] for an example using polarized bremsstrahlung), the measurement of the azimuthal angle will allow us to gain in-

*e-mail: mpeck@ikp.tu-darmstadt.de formation about the structure of intermediate states and the path through the fission barrier. The position-sensitive detector was designed and constructed by a collaboration of TU Darmstadt, European Commission's Joint Research Centre Geel and GSI Helmholtzzentrum für Schwerionenforschung $\mathrm{GmbH}$, based on the detector by Göök et al. [3]. Upon assembly, the new ionisation chamber has been tested in an experiment at JRC Geel using a ${ }^{252} \mathrm{Cf}$ source. Results presented in this manuscript originate from this commissioning experiment.

\section{Experimental set-up}

\subsection{Ionization chamber}

In order to achieve position-sensitivity, the anode plates in a standard twin FGIC were replaced by an array of gridand strip-anodes. A common cathode holding the radioactive, spectroscopic ${ }^{252} \mathrm{Cf}$ sample is dividing the detector in two mirror-inverted chamber parts. On the left side of Fig. 1 a schematic illustration of the assembled positionsensitive FGIC is depicted, the distance between cathode and Frisch-grid is $D=35 \mathrm{~mm}$ and the distances between Frisch-grid and grid-anode and grid-anode and strip-anode are $d=4 \mathrm{~mm}$, respectively. The distances were optimized to investigate the position-sensitive structure using heavy ions, alpha particles emitted at the ${ }^{252} \mathrm{Cf}$ sample are not fully stopped between the cathode and Frisch-grid. The Frisch-grid tungsten wires have a diameter of $0.05 \mathrm{~mm}$ and are arranged with a period of $1.0 \mathrm{~mm}$. The chamber is operated using P-10 gas $\left(90 \% \mathrm{Ar}+10 \% \mathrm{CH}_{4}\right)$ at a pressure of 
$1.022(5) \cdot 10^{5} \mathrm{~Pa}$ and a constant gas flow of $50 \mathrm{mln} / \mathrm{min}^{1}$. The reduced field strength in the region between cathode and Frisch-grid is chosen to guarantee a constant electron drift velocity and to minimize electron-ion recombination in the gas. Applied voltages of $\mathrm{U}_{\mathrm{C}}=-2.225 \mathrm{kV}$ and $\mathrm{U}_{\mathrm{G}}=-0.5 \mathrm{kV}$ result in a reduced field strength of $0.48 \mathrm{kV} /\left(\mathrm{cm} \cdot 10^{5} \mathrm{~Pa}\right)$. Furthermore, to minimize signalloss at the Frisch-grid and the grid-anode, the electric fields between Frisch-grid, grid-anode and strip-anode are chosen to be of increasing strength. Analog to Ref. [3], a Frisch-grid to grid-anode field strength three times that between cathode and Frisch-grid was applied, while between grid-anode and strip-anode a field strength twice that between Frisch-grid and grid-anode was applied $\left(\mathrm{U}_{\mathrm{GA}}=+0.1 \mathrm{kV}, \mathrm{U}_{\mathrm{SA}}=+1.3 \mathrm{kV}\right)$.

To investigate the functionality of the position-sensitive structure, a circular ${ }^{252} \mathrm{Cf}$ sample, deposited on a thin transparent backing $\left(220 \mu \mathrm{g} / \mathrm{cm}^{2} \mathrm{Ni}\right)$, allowing a simultaneous measurement of the two fission fragments, was placed in the central hole of the common cathode. As fission fragments emitted in ${ }^{252} \mathrm{Cf}(\mathrm{sf})$ are well studied and properties like fission-fragment masses and total kinetic energies (TKE) well known [6], a reliable characterization of the detector is possible.

\subsection{Data acquisition}

Figure 1 includes a schematic illustration of the data acquisition system. Analog signals collected at the electrode plates $[7,8]$ are processed using a waveform digitiser. The cathode signal triggers a gate, in which data is buffered to the digitiser. The waveform data were stored and processed offline with baseline corrections and high- and lowpass filters to extract the respective pulse-height and timing information used to determine fission-fragment mass and TKE distributions as well as polar angular distributions.

\subsection{Position-sensitive electrodes}

Two anode plates per chamber side were installed to achieve position-sensitivity: a grid-anode and a stripanode. The sensitive array of the grid-anode consists of 50 tungsten wires with a radius of $0.025 \mathrm{~mm}$ and a spacing of $2.0 \mathrm{~mm}$. The wires are installed on a circular printed circuit board (PCB), strained over a quadratic $100 \mathrm{~mm} \mathrm{x}$ $100 \mathrm{~mm}$ opening, see Fig. 2. All wires are electrically insulated and are connected via $100 \Omega$ surface-mount resistors, creating a resistive charge-divider with 51 resistors in total. The choice of resistance of the charge dividing structure needs to balance two main effects [4] and is discussed in detail in Ref. [3]. On both ends of the anode plate preamplifiers are connected, and waveform data is collected. The strip-anode is manufactured similar to the grid-anode: Instead of wires, 50 parallel, electrically insulated strips are placed on a PCB. Again, $100 \Omega$ surface-mount resistors connect the strips and create a resistive charge-divider. To

\footnotetext{
${ }^{1} \mathrm{ml}$ normal per minute: reference conditions are a temperature of $0^{\circ} \mathrm{C}$ and a pressure of $1.013 \cdot 10^{5} \mathrm{~Pa}$.
}

obtain 2D-position-sensitivity, the grid- and strip-anode on one chamber side have to be installed in such a way that grids and strips are arranged perpendicularly to each other.

\section{Acquired data and signal treatment}

Analog signals collected at the electrode plates due to drifting electrons in the applied electric field are processed using a waveform digitiser ${ }^{2}$. The cathode signal is used to open a gate in which data is buffered to the digitiser, see Fig. 1 for a schematic illustration of the data-acquisition chain. The waveform data were stored and processed offline to extract pulse-height and timing information used to determine fission-fragment mass and TKE distributions as well as polar and azimuthal angular distributions. A detailed description of the waveform analysis can be found in Ref. [3]. The induced and collected charge on the gridand strip-anode, respectively, is divided between the near (n) and far (f) end pre-amplifiers. The integrals of the induced charge signals from the grid-anode pre-amplifiers $P_{n, f}$ and the maxima of the collected charge signals from the strip-anode pre-amplifiers $A_{n, f}$ are used to introduce coordinates according to

$$
\bar{x}=k_{x} \frac{P_{n}-P_{f}}{P_{n}+P_{f}}=k_{x} X, \quad \bar{y}=k_{y} \frac{A_{n}-A_{f}}{A_{n}+A_{f}}=k_{y} Y,
$$

where $k_{x}$ and $k_{y}$ are calibration constants relating the pulse height ratios $X$ and $Y$ to actual distances from the center of the detector. The third Cartesian coordinate $\bar{z}$ is extracted from the average electron drift time $\bar{t}$ according to

$$
\bar{z}=v_{d}\left(\bar{t}_{0}-\bar{t}\right)
$$

where $v_{d}$ is the electron drift velocity in the counting gas, and $\bar{t}_{0}$ corresponds to $\bar{t}$ for events emitted parallel to the target plane and, hence, with maximum drift time values.

\subsection{Mass and TKE distributions}

In order to make sure that the energy and mass resolution compared to a standard Frisch-grid ionization chamber (e.g., Ref. [1]) is not affected by exchanging the anode plates with segmented ones, mass and total kinetic energy distributions of fission fragments emitted in ${ }^{252} \mathrm{Cf}(\mathrm{sf})$ have been determined. Figure 3 shows a pre-neutron mass distribution obtained with the position-sensitive detector in comparison to results of Göök et al. [9] taken with a standard ionization chamber. To correct for evaporation of neutrons during the fission process, data from Ref. [9] has been used. The pulse-height defect in the counting gas, an effect that causes a non-linear response of the pulse height with respect to energy, has been taken into account as stated in Ref. [10]. The excellent agreement in shape of the two distributions ensures that the energy and mass resolution for fission fragments is not affected by the position-sensitive structure.

\footnotetext{
${ }^{2}$ Teledyne SP Devices, model ADQ214 - Dual, 14-bit, 400 MS/s.
} 


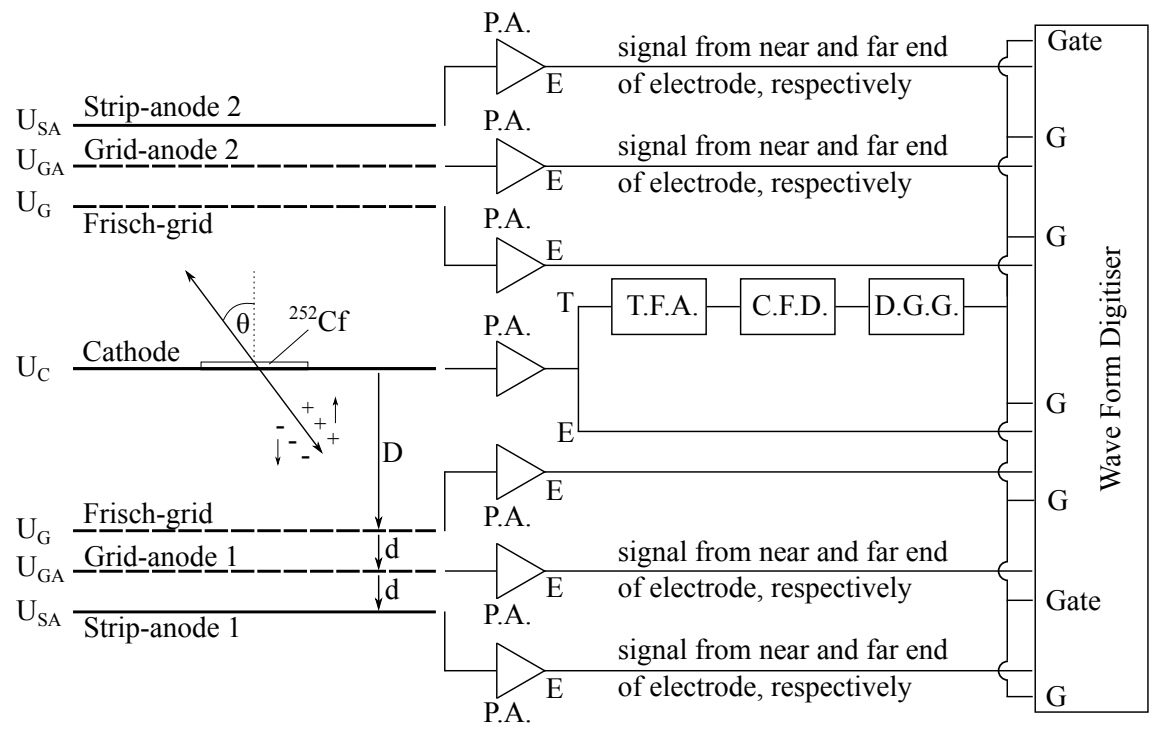

Figure 1. Schematic illustration of the Frisch-gridded position-sensitive ionization chamber and the connected data acquisition. For simplicity, only one signal path at each strip- and grid-anode is depicted, in total 11 channels of the wave form digitiser had to be used. The respective abbreviations are: P.A. - Pre-Amplifier, T.F.A. - Timing Filter Amplifier, C.F.D. - Constant Fraction Discriminator, D.G.G - Digital Gate Generator.

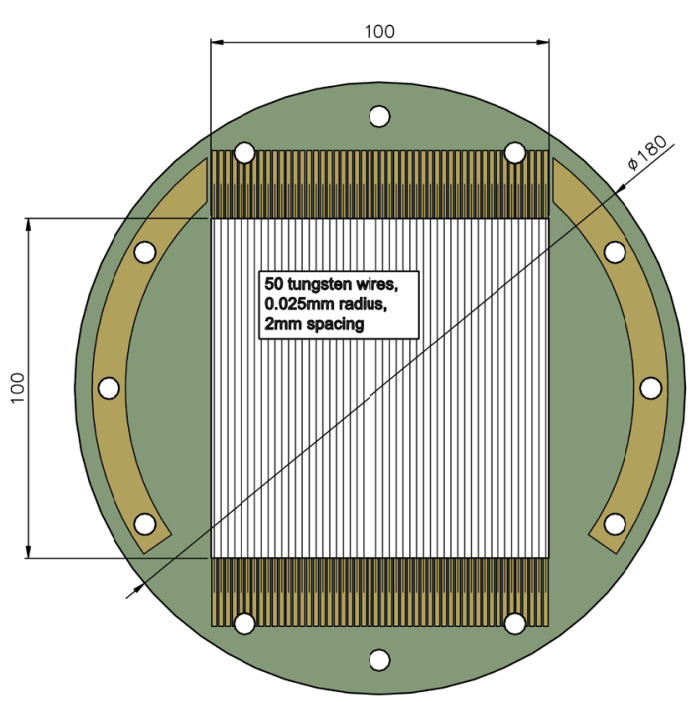

Figure 2. Technical drawing of the position-sensitive gridanode. A centric $100 \mathrm{~mm} \times 100 \mathrm{~mm}$ opening on the PCB (printed circuit board) is covered by 50 tungsten wires with a radius of $0.025 \mathrm{~mm}$ and a period of $2.0 \mathrm{~mm}$. The wires are connected via 51 surface-mount resistors.

\subsection{Extraction of position information}

From the position-sensitive grid- and strip-anodes and the assumption of fission-fragments collinearity, the orientation of the fission axis is recalculated by determining two points in space, see Ref. [3]. In the present gas-filled detector these two points correspond to the center of gravity of the two ionization tracks created by the fission fragments in the counting gas. The coordinate system used to describe the fissioning system is defined in Ref. [3],

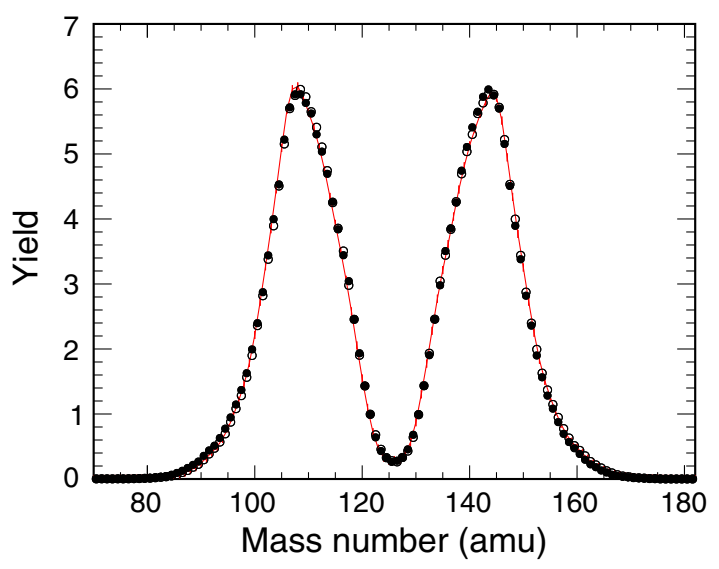

Figure 3. Calculated pre-neutron mass yield compared to established data from Göök et al. [9] (full red line). Full circles: data measured at the side directed to the sample backing, open circles: data measured at the side directed to the sample active side.

as well. The fission origin is fixed to the target plane $\left(x_{0}, y_{0}, 0\right)$, and the fission axis orientation can be expressed either in Cartesian coordinates $(\bar{x}, \bar{y}, \bar{z})$ or by polar and azimuthal angles $(\theta, \phi)$. By measuring the electron drift time, the polar angle is calculated in terms of $\cos \theta$ for each chamber side, respectively, see Ref. [2]. The azimuthal angle is determined using the collinearity of the fission fragments. The differences of the $\bar{x}$ - and $\bar{y}$-coordinates from both chamber sides $\left(\bar{x}_{i}, \bar{y}_{i}, i=1,2\right)$ are independent of the point of creation of the fission fragments. The azimuthal angle is calculated by

$$
\phi=\arctan \left[\frac{\bar{y}_{2}-\bar{y}_{1}}{\bar{x}_{2}-\bar{x}_{1}}\right] .
$$




\subsection{Relative calibration of coordinates}

In order to obtain accurate Cartesian coordinates $(\bar{x}, \bar{y}, \bar{z})$ describing the fission axis, the $\bar{x}$ - and $\bar{y}$-coordinates calculated using Eq. (1) and the $\bar{z}$-coordinate calculated using the electron drift time measurement described by Eq. (2) have to be calibrated relative to each other. A non-linearity in the $\bar{x}$ - and $\bar{y}$-coordinates exists, which does not depend on the absolute value of $\bar{x}, \bar{y}$ from either chamber side, but only on the polar angle $\theta$, see Ref. [3]. To correct for the non-linearity and to calibrate $\bar{x}$ and $\bar{y}$ relative to $\bar{z}$, the difference in pulse-height ratio $X_{2}-X_{1}$ and $Y_{2}-Y_{1}$ divided by the sum of distances to the center of gravity of the ionization track $\bar{r}_{1}+\bar{r}_{2}$ for each chamber side, respectively, is calculated for several values of $\cos \theta$. Figure 4 shows an example of a histogram for a $\cos \theta$-value of 0.2. To each selection in $\cos \theta$ an ellipse with semi-axis $r_{x}$ and $r_{y}$ is fitted. The calibration constants $k_{x}$ and $k_{y}$ introduced in Eq. (1) are functions of $\cos \theta$ and are related to the semi-axis via

$$
r_{x}=\frac{\sqrt{1-\cos ^{2} \theta}}{k_{x}(\cos \theta)}, \quad r_{y}=\frac{\sqrt{1-\cos ^{2} \theta}}{k_{y}(\cos \theta)} .
$$

The accuracy of the $\bar{x}$ - and $\bar{y}$-coordinates is related to the width of ellipses like shown in Fig. 4. By determining the width of the ellipses and correcting for the contribution of the finite resolution in $\cos \theta$ the accuracy of the $\bar{x}$ - and $\bar{y}$ coordinates is estimated. The mean accuracy determined in this work agrees well with the resolution derived in Ref. [3]. Both detectors resolve the determined fission axis with a resolution better than $7^{\circ} \mathrm{FWHM}$.

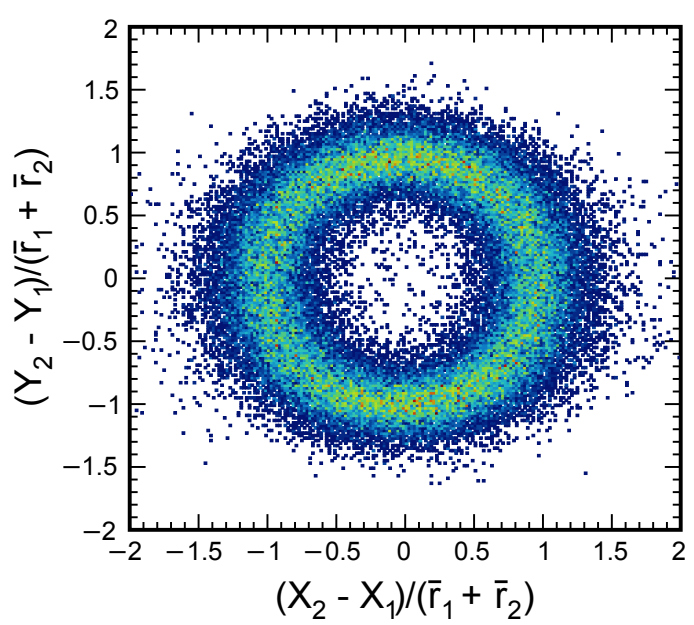

Figure 4. Difference in pulse height ratio $X_{2}-X_{1}$ and $Y_{2}-Y_{1}$ divided by the sum of distances to the center of gravity of the ionization track $\bar{r}_{1}+\bar{r}_{2}$ for each chamber side plotted for a $\cos \theta$ value of 0.2 .

\section{Conclusion and Outlook}

A twin position-sensitive Frisch-grid ionization chamber has been set up and tested successfully using the wellknown ${ }^{252} \mathrm{Cf}$ spontaneous fission decay. By exchanging the standard anode plates in the FGIG by a positionsensitive structure the excellent energy and mass resolution for fission fragments was not affected. The position information of the center of gravity of the ionization tracks was used for the determination of the fission axis relative to an arbitrary axis in space with a resolution better than $7^{\circ}$ FWHM.

The described detector will be used in photofission experiments on actinides at excitation energies in the barrier and the GDR region. Correlated mass, total kinetic energy and angular distributions (see Ref. [13]) of fission fragments will be for the first time accessible for study with quasimonochromatic, polarized $\gamma$-rays.

\section{Acknowledgements}

M. Peck thanks the EC-JRC in Geel, Belgium, for the hospitality during the measurements for this study. Financial support through the German BMBF [grant no. 05P18RDEN9] and the Hessian HMWK through the LOEWE research center Nuclear Photonics is gratefully acknowledged.

\section{References}

[1] C. Budtz-Jørgensen, H.-H. Knitter, C. Straede, F.-J. Hambsch, R. Vogt, NIMA 258 (1987) $209-220$.

[2] A. Göök, M. Chernykh, J. Enders, A. Oberstedt, S. Oberstedt, NIMA 621 (2010) $401-405$.

[3] A. Göök, W. Geerts, F.-J. Hambsch, S. Oberstedt, M. Vidali, Sh. Zeynalov, NIMA 830 (2016) $366-374$.

[4] A. Pullia, W.F.J. Muller, C. Boiano, R. Bassini, IEEE Trans. Nucl. Sci., 49 (2002) 3269 - 3277.

[5] R. Ratzek, W. Wilke, J. Drexler et al., Z. Phys. A 308 (1982) $63-71$.

[6] H. Henschel, A. Kohnle, H. Hipp, G. Gönnenwein, NIM 190 (1981) 125 - 134.

[7] W. Shockley, J. Appl. Phys. 9 (1938) $635-636$.

[8] S. Ramo, Proc. I.R.E. 27 (1939) $584-585$.

[9] A. Göök, F.-J. Hambsch, M. Vidali, Phys. Rev. C 90 (2014) 064611.

[10] M. Peck, A. Göök, J. Enders, F.-J. Hambsch, S. Oberstedt, NIMA 919 (2019) $105-112$.

[11] R. Schmidt, H. Henschel, Nucl. Phys. A 395 (1983) $15-28$.

[12] F. Gönnenwein, in: C. Wagemans (Ed.), The Nuclear Fission Process, CRC Press, Boca Raton, 1991, p. 323.

[13] A. Göök, C. Eckardt, J. Enders, M. Freudenberger, A. Oberstedt, S. Oberstedt, Phys. Rev. C 96 (2017) 044301. 